\title{
La transition vers une Gouvernance démocratique en Guinée
}

\author{
Dominique Bangoura \\ Professeur au Centre d'Etudes Diplomatiques et Stratégiques à Paris et \\ enseignant-chercheur HDR à l'université d'Abidjan
}

\section{Introduction}

La gouvernance du secteur de la sécurité est un domaine mal connu en Guinée. Sur le terrain, ce sujet est encore tabou car largement réservé aux militaires, aux policiers, aux hommes en uniforme et au chef de l'Etat. Les raisons qui permettent d'expliquer cette situation sont historiques et politiques: elles sont liées aux différents régimes qui se sont succédé en Guinée: la dictature civile de Sékou Touré qui a dirigé le pays de l'indépendance du pays à sa mort (1958 à 1984); le régime militaire (1984 à 1990) puis autoritaire (1990 à 2008) du général-président Lansana Conté; et enfin la dictature militaire du capitaine Moussa Dadis Camara, qui a pris le pouvoir le 23 décembre 2008 et l’a exercé jusqu'au 3 décembre 2009, date d’une tentative d’assassinat qui l’a écarté du pouvoir.

Dans ce contexte, les conditions de changement sont difficiles à réunir, tant au plan institutionnel que non-gouvernemental. Il faut attendre début 2010 avec l'accession au pouvoir du général Sékouba Konaté, pour qu’un cadre politique plus favorable apparaisse et pour que les premières mesures politiques et sécuritaires soient prises en matière de gouvernance et de réforme du secteur de la sécurité. Pour la première fois également, le poids et les revendications de la société civile sont pris en compte dans ce processus.

Cependant, ce n'est qu'après la fin de la transition marquée par lélection du président Alpha Condé en novembre 2010 que la gouvernance du secteur de

Comment citer ce chapitre du livre:

Bangoura, D. 2015. La transition vers une Gouvernance démocratique en Guinée.

Dans: Bryden, A et Chappuis, F (dir. publ.) Gouvernance du secteur de la

Sécurité : Leçons des expériences ouest-africaines, Pp. 41-63. London: Ubiquity Press. DOI: http://dx.doi.org/10.5334/bav.c. Licence: CC-BY 4.0. 
la sécurité prend tout son sens, d'autant plus qu'il existe une volonté politique forte au sommet de l'Etat et une grande motivation de poursuivre ces efforts de la part des différents acteurs étatiques et non-étatiques. La question qui se pose maintenant est de savoir si ces promesses seront tenues durant le mandat de l'actuel président.

\section{Contexte de la gouvernance du secteur de la sécurité en Guinée}

La place et le rôle de l'armée et des forces de sécurité ont varié d'un régime à l'autre. Sékou Touré, qui est un leader civil, instaure et développe un autoritarisme dur teinté d'idéologie marxiste en utilisant et en instrumentalisant l’armée dont il se méfie: il préfère s'appuyer sur la Milice, la branche armée du parti unique, totalement acquise à sa cause. Lansana Conté, qui est un chef militaire avant tout, fait de l'armée l'un des principaux piliers de son accès et de son maintien au pouvoir. Moussa Dadis Camara, qui arrive au pouvoir par un coup d'Etat militaire, utilise très rapidement les forces armées pour asseoir son régime et mater l'opposition politique ainsi que les civils.

\section{La gouvernance du secteur de la sécurité sous Sékou Touré (1958-1984)}

La Guinée est le premier pays francophone d'Afrique de l'Ouest à obtenir son indépendance dès le 2 octobre 1958, quelques jours après le "non" historique au référendum du 28 septembre proposé par le général de Gaulle. ${ }^{1}$

L'armée, une force mal lotie et politisée. L'armée guinéenne est créée le 1er novembre 1958. Elle est constituée de volontaires issus de larmée coloniale (Horoya 1993a; 1993b). Toutefois, tous les soldats guinéens ne sont pas autorisés à entrer dans la jeune armée nationale : une partie se voit refuser l'intégration pour des raisons politiques (Bah 2009). ${ }^{2}$ A ses débuts, l'armée guinéenne se fait connaitre sur le continent africain par son contingent qui intègre les Casques bleus onusiens au Congo et par son engagement aux côtés des mouvements de libération nationale (Angola, Mozambique, ${ }^{2}$ etc.) (Soumah 2004: 162). Toutefois, sous Sékou Touré, larmée guinéenne manque de moyens, de formation et d'équipement; certains sous-officiers et officiers sont formés dans les pays d'Europe de l'Est et à Cuba. L'armée reçoit du matériel soviétique, mais elle est mal lotie et mal organisée.

Deux raisons expliquent les difficultés auxquelles est exposée l'armée. Tout d'abord, Sékou Touré se méfie des militaires, responsables sur le continent africain de coups d'Etat qui ont renversé plusieurs chefs d'Etat civils. ${ }^{3}$ Le premier président guinéen préfère concentrer tous les moyens au profit du parti unique qu'il a créé, le Parti démocratique de Guinée (PDG). Selon le journaliste et homme politique guinéen, Siradiou Diallo, «le parti, conçu 
comme l'institution dirigeante, le centre moteur et l'instrument de contrôle de la vie nationale, est placé au-dessus de la pyramide étatique. Toutes les autres institutions, en l'occurrence l'armée et la milice lui sont subordonnées et ne représentent que des organes d'exécution, des instruments au service du parti.» (Diallo 1986). C'est ainsi que le PDG est qualifié de parti-Etat, ce qui illustre sa logique omniprésente (Bah 2009) et totalitaire (Camara 1998: 64). ${ }^{4}$

Le régime au pouvoir surveille donc étroitement l'armée. ${ }^{5}$ En outre, l'armée est fortement soumise au parti, le PDG. Cette conception de l'armée est lourde de conséquences. La politisation de l'armée entraîne l'indiscipline car elle permet à un simple caporal politisé, zélé et proche du pouvoir, de discuter l'ordre d'un supérieur hiérarchique, voire de l'humilier. Ce non-respect de la hiérarchie est source de dysfonctionnements car il banalise le commandement militaire aux yeux des subordonnés. De plus, la politisation de larmée aura des conséquences par la suite: elle encouragera les militaires à prendre le pouvoir (coups d'Etat de 1984 et de 2008) et à le contester (tentatives de contre-coups d'Etat et mutineries à répétition sous Lansana Conté, tentative d'assassinat de Moussa Dadis Camara).

La Milice, une force de contrôle au-dessus de l'armée. Sous Sékou Touré, la milice populaire est une force paramilitaire puissante, au-dessus de l'armée. Elle est conçue comme un bouclier de protection, de sécurité rapprochée du chef de l'Etat. Elle relève directement de son autorité et elle est, avec le parti unique, l'un des principaux instruments de domination. Elle a théoriquement pour mission d'assurer le maintien de l'ordre aux côtés de la police mais, en réalité, elle est chargée de surveiller l'armée et de contrôler la population. C'est une police politique armée qui excelle dans la filature, la délation et les arrestations arbitraires. Les grades dans la milice sont les mêmes que dans l'armée. Les miliciens commettent fréquemment des exactions à l'encontre des policiers, gendarmes et militaires. Cette structure parallèle provoque l'hostilité des militaires de carrière qui réagissent en paroles, parfois en actes, mais sans pouvoir la remettre en cause. Vers la fin de sa vie, le président Sékou Touré dispose d'une milice dont les effectifs contrebalancent ceux de l'armée: 10000 hommes de chaque côté. Cependant, la milice est bien mieux considérée.

Dans un tel régime, aucune force sociale ou politique ne peut exercer la moindre surveillance ou le moindre contrôle en dehors des structures du PDG. Sékou Touré meurt brutalement, le 26 mars 1984, d'une attaque cardiovasculaire, laissant le pays exsangue et des prisons surpeuplées, sans oublier les innombrables morts décédés sous la torture et les disparus. Le nombre exact des victimes de son régime n’a jamais été officiellement connu, faute de recensement et d'enquête indépendante. Quelques jours plus tard, le 3 avril 1984, l'armée prend le pouvoir avec à sa tête Lansana Conté. Après 26 ans de dictature civile, le pays entre dans lère de la dictature militaire. 


\section{La gouvernance du secteur de la sécurité sous Lansana Conté} (1984-2008)

Le Général Lansana Conté se maintient 24 ans au pouvoir. Ainsi, de 1958 à 2008, la Guinée ne connaîtra que deux chefs d'Etat, l'un civil, l'autre militaire, mais tous deux ont en commun de gouverner par la force et la violence.

Les trois périodes de la gouvernance de Lansana Conté. On peut distinguer trois périodes dans l'exercice du pouvoir par Lansana Conté. La première, de 1984 à 1990, est celle du régime militaire. Celui-ci procède à la dissolution du parti unique (PDG) et suspend la Constitution de 1982, mais n'engage aucune réforme. C'est un régime d'exception. La deuxième, qui dure de 1990 à 1995, est celle de l'instauration d'un Etat de droit avec l'adoption de la Constitution de décembre 1990 par référendum. C'est la période de libéralisation du régime avec l'adoption du multipartisme et une ouverture sur les libertés publiques. Néanmoins, cette période est de courte durée. Des élections présidentielle (1993) et législatives (1995) sont organisées et remportées par le pouvoir en place; mais ces scrutins provoquent de vives contestations du fait de fraudes massives. La troisième période est celle qui sétend de 1996 à 2008. En février 1996, Lansana Conté est menacé de renversement, à l'occasion d'une mutinerie qui éclate dans l'armée pour des raisons corporatistes; le chef de l'Etat y met un terme en acceptant toutes les revendications, mais il fait arrêter de nombreux militaires. A partir de là, le régime sombre dans la restauration autoritaire. En novembre 2001, un référendum constitutionnel supprime la limitation des mandats présidentiels et permet ainsi à Lansana Conté de se représenter indéfiniment. De fait, en 1998, le président est candidat à sa propre succession (tandis que le principal candidat de l'opposition, Alpha Condé, est arrêté et emprisonné). Il en est de même en 2003. Lors de ces deux élections, le chef de l'Etat remporte le scrutin tandis que l'opposition crie à la fraude. Quelques mois auparavant, en 2002, dans des circonstances similaires, les législatives sont remportées par la majorité au pouvoir, l'opposition ayant choisi de les boycotter, à l'exception de trois partis.

Dans un tel contexte, il n'y a pas ou très peu de place pour des forces démocratiques de surveillance ou de contrôle. L'Assemblée nationale est considérée comme une chambre d'enregistrement, acquise à la cause du régime en place. Sur le plan sociopolitique, le pouvoir n'hésite pas à recourir à une répression sanglante pour mater à plusieurs reprises les revendications sociales et politiques des syndicats et des forces vives qui revendiquent le changement (février-mars et juin 2006, janvier 2007).

Les divisions dans l'armée et la rivalité avec les forces de sécurité. Larmée est divisée. Elle souffre de conflits de génération, de tensions ethniques et de rivalités personnelles, si bien que les différents groupes d'officiers se neutralisent les uns les autres. Elle recrute sur des critères politiques et ethniques et elle est peu formée, mal entraînée; les promotions militaires sont accordées 
non pas selon la compétence mais en fonction de l'allégeance au pouvoir. Elle présente d'énormes disparités entre la haute hiérarchie proche du chef de l'Etat qui évolue dans l'opulence et la troupe qui vit dans la misère. De ce fait, l'indiscipline, qui avait commencé sous Sékou Touré, continue de régner dans les casernes.

De plus, un climat de rivalité et de méfiance règne entre forces de défense et forces de sécurité. C’est le cas entre l'armée et la police car sous Sékou Touré, larmée était contrôlée par la milice, la police politique. En tant que militaire, Lansana Conté accorde plus d'attention et de moyens à la gendarmerie quà la police, une force de sécurité chargée théoriquement de la protection des personnes et des biens. Le rôle et le statut des forces de police restent marginaux et, à plusieurs reprises, notamment en juin 2008, la mauvaise perception de la police par l'armée et la gendarmerie aboutit même à la destruction d'un commissariat de police et à des affrontements. Ainsi, le 17 juin 2008, des militaires répriment dans le sang des policiers qui revendiquent une hausse de salaire et des primes.

\section{La gouvernance du secteur de la sécurité sous Moussa Dadis Camara (2008-2009)}

Le président Conté est officiellement décédé dans la soirée du 22 décembre 2008. Lannonce a été faite sur les ondes de la télévision nationale vers une heure du matin par Aboubacar Somparé, le président de l'Assemblée nationale; cette information est relayée par le premier ministre Souaré et le chef d'état-major des armées, le général Diarra Camara. Cette procédure a laissé penser durant quelques heures que le processus de succession constitutionnelle, prévu par l'article 34 de la Constitution, était amorcé. Cependant, dès le lendemain matin, le 23 décembre vers $7 \mathrm{~h} 30$, des putschistes lisent à la radio guinéenne leur première déclaration. ${ }^{6}$ Le porte-parole, le capitaine Moussa Dadis Camara, annonce la suspension de la constitution, des institutions de l'Etat, de la vie politique et syndicale ainsi que la création d'un comité militaire, le Conseil national pour le développement et la démocratie (CNDD). Le 24 au soir, il s’autoproclame président de la République.

Le capitaine Camara ouvre de lui-même une transition politique, dont les enjeux sont théoriquement clairs: réunir les conditions permettant à la Guinée dorganiser des élections libres et transparentes en vue de se doter d'un régime politique démocratique via une alternance (Bangoura, Bangoura \& Diop 2006). Il promet qu'il ne sera pas candidat à sa propre succession.

Cependant, dans les faits, la situation ne tarde pas à susciter des craintes. Le gouvernement, nommé par décret le 14 janvier 2009, est largement dominé par les militaires. ${ }^{7}$ Le discours-programme du 14 janvier 2009 sapparente à un 
vaste chantier; or, la transition ne doit durer que quelques mois. Le capitaine Camara n'a ni le temps ni la légitimé pour mener une action d'une telle envergure d'autant plus que les bailleurs de fonds ont condamné le putsch.

La gouvernance du chef de la junte est faite de voltefaces, de signaux contradictoires, de populisme, de colères au cours desquelles il destitue des cadres en public et s'en prend à un diplomate occidental. Aucune institution de la transition n'est mise en place. Cette période de transition reçoit le coup de grâce lors de la sixième session du Groupe international de contact pour la Guinée (GIC-G), les 3 et 4 septembre 2009 à Conakry. A cette date, revenant à la charge et faisant voler en éclats tout espoir d'apaisement, Moussa Dadis Camara rompt son serment. En aparté, il glisse aux diplomates présents : «Je vais me présenter parce que tout le peuple me le demande».

Cette première période de transition est un échec. Elle se termine par les massacres perpétrés au stade de Conakry, le 28 septembre 2009, par les forces de défense et de sécurité du régime contre des militants des partis politiques qui réclament un processus électoral ouvert et démocratique. Autre fait grave: le 3 décembre 2009, le capitaine Camara est lui-même victime d'une tentative d'assassinat par le chef de sa garde rapprochée. Cet acte est consécutif au refus de Toumba Diakité d’aller répondre, comme le lui demande le président, à l'interrogatoire de la Commission internationale d'enquête de l'ONU sur les massacres perpétrés le 28 septembre 2009. Toumba Diakité a-t-il agi par crainte de poursuites judicaires ou parce que, par principe, il estimait que la responsabilité de ces actes relevait du chef de l'Etat? Cette situation montre une fois encore jusqu'où peut aller la violence et l'indiscipline au sein des forces armées et de sécurité en Guinée.

En conclusion, force est de constater qu'en Guinée, depuis l'indépendance, l’armée, la gendarmerie et la police (y compris la milice sous Sékou Touré) se sont illustrées par des actes de violence et des exactions répétées contre les populations civiles et contre les citoyens. Ces forces laissent apparaître de profonds dysfonctionnements, une forte politisation et un manque de discipline récurrents. Cette situation s'explique principalement par la nature des régimes politiques au pouvoir: de 1958 à 2010, la Guinée n'a connu aucun régime démocratique. Dans un tel contexte, il est indispensable de pouvoir procéder à des réformes en profondeur de la gouvernance et du secteur de la sécurité. Dans cette perspective, il importe d'identifier les conditions permettant un tel changement; les défis à relever ou les obstacles à surmonter; les efforts à encourager; ou les dynamiques de transformation à soutenir.

\section{Les conditions du changement}

Quelles sont les conditions pour que la situation politique, militaire et sécuritaire change? Quels sont les freins et les facteurs favorables? 


\section{Létat des systèmes administratif, législatif et judiciaire}

La question qui se pose est celle du rôle potentiel ou réel du système administratif, du pouvoir législatif et du pouvoir judiciaire dans la transformation ou le changement dans la gouvernance du secteur de la sécurité. Quelles sont les possibilités à exploiter pour que la séparation des pouvoirs soit effective et pour que la justice devienne indépendante?

En Guinée, il est bien connu que l'administration est un rouage de l'Etat et en particulier un pilier du régime au pouvoir. Cela sest vérifié sous chaque gouvernement, depuis l'indépendance du pays. Ladministration n'est pas un corps neutre; elle est au service du pouvoir exécutif. Par exemple, sous Lansana Conté, les élections étaient organisées par le gouvernement, en particulier par les ministères concernés (Administration du territoire et de la Décentralisation, Intérieur) en concertation avec la Commission nationale électorale (1993), le Haut-Conseil chargé des affaires électorales (1998) ou la Commission électorale nationale autonome (2005), qui nétaient pas des organes indépendants. Il a fallu attendre mai 2007 pour qu’un projet de loi portant création d'une Commission électorale nationale indépendante (CENI) soit voté par l'Assemblée nationale (Bangoura 2007: 97). Cette CENI était un facteur positif de changement parce que, sur la forme, elle avait été le fruit d'un long et difficile dialogue entre les partis politiques (de la mouvance présidentielle et de l'opposition) et le gouvernement. Sur le fond, la CENI était une nouvelle institution censée pouvoir organiser et réguler le futur processus électoral.

A vrai dire, la création de la CENI par l'Assemblée nationale peut être considérée comme un exploit car, à cette période, la composition de l'Assemblée nationale ne reflétait pas l'ensemble de la classe politique. Pour mémoire, des élections législatives avaient eu lieu en 2002, mais elles avaient été boycottées par la plupart des partis de l'opposition, mis à part l'Union pour le Progrès et le Renouveau, l'Union pour le Progrès de la Guinée, et le Parti du Peuple de Guinée. Concomitamment à la création de la CENI, l'Assemblée avait voté, lors de sa première session en mai 2007, la loi portant modification du code électoral, la loi de financement des partis politiques et la loi portant statut des partis de lopposition. Elle avait également voté la loi portant création de la Commission nationale d'enquête sur les massacres de juin 2006 et de janvierfévrier 2007. ${ }^{8}$ Les facteurs ayant contribué à ce vote étaient sans doute, en amont, le poids des revendications démocratiques portées par la société civile et les syndicats au cours des événements de 2006 et de 2007, revendications qui ont abouti à l'Accord tripartite du 27 janvier 2007, et à la nomination d'un premier ministre de consensus, Lasana Kouyaté, chef de gouvernement en application de cet accord. ${ }^{9}$

Cependant, en dehors de ces lois qui ont été très importantes pour le processus démocratique, l'Assemblée nationale n’a pas été en mesure, dans les sessions ultérieures de septembre 2007 et de 2008, de se pencher sur les questions 
de défense et de sécurité, qui étaient encore des domaines réservés du chef de l'Etat, le général Lansana Conté. Bien que la Constitution (article 59) et le Règlement intérieur de l'Assemblée nationale le permettent, cette dernière ne jouissait pas encore d'une culture démocratique et d'une indépendance suffisantes pour exercer un contrôle sur le gouvernement.

La justice n'est pas davantage indépendante. Selon l'avocat guinéen maitre Thidiane Kaba, sous Lansana Conté, «l'asservissement du système judiciaire au pouvoir exécutif ne permet pas d'assurer une bonne administration de la justice» (Kaba 2007: 119), d’autant plus que ce secteur est miné par la corruption. Le problème n'est pas l'absence de cadre institutionnel car la loi fondamentale de décembre 1990 prévoit un Etat de droit démocratique, y compris l'indépendance et le bon fonctionnement de la justice. Les carences résident principalement dans la non-application de la constitution, le premier à violer la loi fondamentale étant souvent le chef de l'Etat, comme, par exemple, lorsqu'il décide d'aller en personne libérer son ami, le richissime homme d'affaires Mamadou Sylla, incarcéré pour détournement de fonds publics.

Les mêmes difficultés se retrouvent sous Moussa Dadis Camara qui concentre tous les pouvoirs entre ses mains: les ministères clés, dont celui de la Justice, sont tenus par des militaires. Pour ce qui est du ministère de la Défense, le principe de subordination des militaires à l'autorité civile n'est pas appliqué puisque la junte est au pouvoir. Quant à l'Assemblée nationale, elle a été dissoute au moment du coup d'Etat. Le capitaine Camara a confié ce rôle au secrétariat permanent du CNDD, chargé de la révision des lois organiques et de la loi fondamentale, de la réforme de la justice et de l’appui à la réforme des forces de défense et de sécurité. Ce secrétariat rattaché à la junte est tenu par un colonel ayant rang de ministre.

Il faut attendre les revendications et les propositions du Forum des Forces vives de Guinée (société civile et partis politiques), réunies au palais du peuple à Conakry le 12 mars 2009, pour pouvoir obtenir, non sans difficulté et à l'issue de longues négociations, le principe d'un parlement de transition dénommé Conseil national de transition (CNT). Concrètement, la mise en place de cet organe pose problème. Un projet d’ordonnance est présenté par une délégation du CNDD en visite à Bruxelles le 24 avril 2009. Ce projet est contesté par les Forces vives pour son manque d'indépendance envers la junte. Une nouvelle version est proposée par le ministre secrétaire permanent du CNDD le 27 avril. C'est sur cette base que les Forces vives négocient avec le CNDD, début mai, un projet de création du CNT précisant son mandat et la répartition de ses membres dont le nombre est fixé à 163. Cependant, par la suite, le texte est modifié unilatéralement par la junte qui porte le nombre de membres à 244 pour y ajouter des individus totalement acquis à sa cause. Cest seulement sous la gouvernance du général Sékouba Konaté, parvenu au pouvoir suite à la nouvelle donne politique (Accord de Ouagadougou), que le CNT sera installé en tant qu'institution de la transition et pourra jouer son rôle. 
Dans ces conditions, on comprend que les systèmes de gouvernance sous Lansana Conté et Moussa Dadis Camara ont constitué des obstacles au changement.

\section{Des mécanismes de surveillance déterminés mais limités}

Néanmoins, sous Lansana Conté, des mécanismes d’observation, de surveillance et de contrôle ont été mis en place grâce, d'une part, à la création en 2008, de l'Observatoire national des droits de l'homme (ONDH) et d'autre part, au rôle croissant des organisations de la société civile.

De l'ONDH à la CNDH. En juin 2008, la Guinée s'est dotée d'un Observatoire national des droits de l'homme, rattaché à la primature. La présidence de cette institution a été confiée à Aliou Barry, docteur en droit international public et professionnel des questions de défense et de sécurité, qui a été nommé par le premier ministre sur la base de ses compétences. La création de l'ONDH intervient quelques mois après les violences de 2006 et de 2007 au cours desquelles les manifestations des syndicats et de la société civile ont été réprimées dans le sang. L'ONDH, chargée de la promotion et du respect des droits de l'homme, bénéficie du soutien des Nations Unies. Cependant, la mise sur pied de cet observatoire n'a pas empêché de nouvelles violations des droits humains et le président de cette institution a lui-même été grièvement blessé par des éléments de l'armée et de la garde présidentielle dans l'exercice de ses fonctions (Conakryka 2010). ${ }^{10}$ Le régime au pouvoir n'a donc pas permis à l'ONDH d'assumer pleinement ses fonctions.

Il a fallu attendre mai 2010 et l'adoption de la nouvelle Constitution de la République de Guinée par le CNT, pour voir l'inscription de l'«Institution nationale indépendante des droits humains» au titre XVI de la loi fondamentale (Guinée 2010: articles 146, 148). ${ }^{11}$ Dans ce prolongement, le président Alpha Condé nouvellement élu, a, par décret du 17 mars 2011, créé la Commission nationale des droits de l'homme (CNDH). ${ }^{12}$ Mamady Kaba, issu de la société civile et précédemment président de la section guinéenne de la Rencontre africaine pour la défense des droits de l'homme a été nommé président de cette commission. Puis, le CNT a voté, le 14 juillet 2011, la loi portant organisation et fonctionnement de de l'«Institution nationale indépendante des droits humains». Ce processus institutionnel en vue d'améliorer la situation des droits de l'homme est un grand pas en avant, du moins sur le plan formel.

Le rôle croissant des organisations de la société civile. La création de la CNDH n’aurait pas été possible sans le rôle soutenu de la société civile qui a mené, en amont, un long plaidoyer. Son action a pu être relayée par les médias. La société civile s'est peu à peu structurée, depuis 2002, pour se fédérer en un Conseil national des organisations de la société civile de Guinée (CNOSCG). Elle s'est distinguée par ses mobilisations de revendications en faveur du changement social et politique en 2006 et 2007. Depuis, elle n'a cessé de lutter contre 
l'impunité et pour le respect des droits de l'homme. Elle a joué un rôle prépondérant de soutien aux victimes des massacres commis en 2006-2007 et en septembre 2009. Elle a permis de réunir des témoignages et des preuves des exactions qui ont été commises et elle coopère avec les organisations internationales (Haut-Commissariat des Nations Unies aux droits de l'homme, Commission internationale d'enquête de l'ONU, Cour pénale internationale, etc.) et les ONG internationales (Fédération internationale des droits de l'homme, Human Rights Watch, Amnesty International, International Crisis Group ...) qui œuvrent dans ce domaine.

En conclusion, une dynamique de changement en faveur de la gouvernance du secteur de la sécurité a été rendue possible grâce à lélaboration et au vote de nouvelles lois; à l'instauration d'une Commission de surveillance et de promotion des droits de l'homme; à la contribution de la société civile au suivi de l'action et du comportement des forces de défense et de sécurité; et au partenariat entre les organisations nationales et internationales de la société civile.

Il apparait que les efforts de la $\mathrm{CNDH}$ et des organisations non-gouvernementales et de la société civile sont à encourager car elles représentent une forme de contre-pouvoir; elles apportent un autre éclairage, une analyse différente des problèmes d'insécurité, d'injustice et de violations des droits de l'homme. Souvent, c'est par le dialogue et par le débat d'idées, les consultations, léchange contradictoire, le suivi et l'observation quelles réussissent à se faire entendre, à devenir une force de proposition. Malgré des faiblesses en termes de moyens de fonctionnement et parfois de représentativité, ces organisations ont tendance à renforcer leurs capacités et leur professionnalisme, ce qui est une garantie de leur durée et de leur efficacité. A titre d’exemple, la société civile est associée à la mise en œuvre d'un projet financé par le Peace Building Fund visant à assurer un contrôle civil et démocratique des forces de défense et de sécurité; ce projet comprend un volet de renforcement des capacités des organisations de la société civile. ${ }^{13}$

Néanmoins, cette dynamique globale n'est pas optimale en raison de plusieurs obstacles dus, d'une part, à la violence politique qui sévit au sommet de l'Etat depuis plus de cinquante ans et d'autre part, au manque de volonté politique de régler pacifiquement le contentieux électoral lié à la préparation des élections législatives. Ces dernières ont été reportées à plusieurs reprises avant de pouvoir se tenir le 28 septembre 2013. De plus, jusquà présent, malgré des progrès dans les relations civilo militaires, la méfiance est encore grande entre les forces de défense et de sécurité (FDS) et la population civile.

\section{Un cadre politique favorable au changement sous la transition du général Sékouba Konaté}

La gouvernance du secteur de la sécurité a connu des améliorations très importantes à partir de janvier 2010. Le contexte de lépoque permet d’en 
comprendre les raisons. La fin de l’année 2009 avait été particulièrement éprouvante pour les Guinéens. Les massacres et viols collectifs commis dans la matinée du 28 septembre 2009 par les forces du régime de Moussa Dadis Camara et la tentative d'assassinat du chef de l'Etat par le commandant de la garde présidentielle le 3 décembre suivant avaient tiré toutes les sonnettes d’alarme. Tant les gouvernés que le principal gouvernant avaient été touchés. Le paroxysme de la violence avait été atteint. Le dysfonctionnement des forces de défense et de sécurité était à son comble.

Tandis que les Forces vives de Guinée pleuraient leurs morts et soignaient les blessés, la Commission internationale d’enquête de l'ONU cherchait à connaitre la vérité sur les massacres du 28 septembre 2009 et à en situer les responsabilités en vue de rendre la justice. Les Forces vives demandèrent la protection de la communauté internationale par le biais d'une force neutre venant s'interposer entre elles et les FDS; elles exigeaient également une réforme en profondeur de l'armée et de la police.

La Déclaration conjointe de Ouagadougou (15 janvier 2010). Un nouveau cadre pour la gouvernance et pour la réforme du secteur de la sécurité (RSS): En l'absence de Moussa Dadis Camara, hospitalisé au Maroc, c'est le n ${ }^{\circ} 2$ de la junte qui gère les affaires courantes à la tête de l'Etat. Il s'agit du général Sékouba Konaté, ministre de la Défense. ${ }^{14}$ Ce dernier décide d’aller rencontrer Moussa Dadis Camara, dès son arrivée en convalescence au Burkina Faso, après l'attentat qui a failli lui coûter la vie. ${ }^{15}$ Les deux hommes se retrouvent les 13 et 14 janvier en compagnie du médiateur, le président burkinabè Blaise Compaoré. A l'issue de leurs entretiens, ils signent la Déclaration conjointe de Ouagadougou, le 15 janvier 2010, par laquelle le général Sékouba Konaté se voit confier officiellement par M. D. Camara l'intérim du pouvoir, pour mener à terme la transition. Cette signature est obtenue sous la pression des Forces vives de Guinée, qui se disent favorables au texte final, bien quelles ne l'aient pas paraphé.

Cet accord crucial comprend trois points: il règle la succession au pouvoir; il fixe les principes et la feuille de route de la transition (une transition de six mois en vue d'organiser une élection présidentielle à laquelle les dirigeants et anciens dirigeants ne seront pas candidats); il fixe la réforme des forces de défense et de sécurité comme une priorité. C'est la première condition réunie pour un début de transformation de la gouvernance du secteur de la sécurité. A partir de là, d'autres facteurs de changement interviennent.

La responsabilisation des FDS par le général Sékouba Konaté. L'une des premières actions du général Sékouba Konaté est de s’adresser aux FDS pour condamner leurs actes de violence et de barbarie perpétrés contre la population et contre leur chef, en l'occurrence le capitaine Moussa Dadis Camara alors chef suprême des armées et aussi pour saluer la mémoire de toutes les victimes. ${ }^{16}$ Son discours du 23 décembre 2009 et ses rencontres dans les casernes sont largement médiatisés et cette campagne de sensibilisation et de responsabilisation des troupes et des officiers est ressentie comme un acte douverture de la part du général et comme une sorte de soulagement dans le pays. 
Lévaluation du secteur de la sécurité par la mission conjointe CEDEAOONU-UA. ${ }^{17}$ Le comportement du président de la transition est salué par la communauté internationale, qui propose alors d’accompagner la Guinée dans les réformes souhaitées par tous. La première est celle de l'évaluation du secteur de la sécurité, par catégories d'acteurs, et aussi dans son ensemble. Ce travail est entrepris et réalisé entre le ler février et le 30 avril 2010 par une mission conjointe placée sous la direction du général Lamine Cissé, un officier sénégalais de grande notoriété tant dans son pays quau sein de la communauté internationale. L'évaluation, qu'il mène avec beaucoup de tact et d'expérience professionnelle, est le point de départ de toute réforme puisquelle dresse létat des lieux du secteur de la sécurité, jusque-là inconnu.

Le rapport dévaluation permet d'établir que le secteur guinéen de la sécurité se trouve dans un état de déliquescence inquiétant et ne respecte aucune norme depuis plusieurs décennies: désorganisation totale des forces de défense et de sécurité; immixtion des militaires dans le domaine politique; absence de contrôle civil; dysfonctionnement du système judiciaire; existence d’acteurs non-étatiques de la sécurité (milices, entreprises privées). Le rapport mentionne la problématique de la circulation des armes légères et de petit calibre dans la région d'Afrique de l'Ouest ainsi que des questions transversales telles que le genre. Il formule ensuite des séries de recommandations sur chacun de ces points, à l'issue d'un processus participatif mené à Conakry et dans plusieurs villes de l'intérieur du pays.

Le rapport est officiellement remis par le chef de la mission conjointe au général Sékouba Konaté, le 4 mai 2010, lors d'une cérémonie au Palais du peuple qui regroupe les principaux acteurs de la vie sociopolitique guinéenne: le gouvernement, le CNT, les partis politiques, la société civile, les acteurs de la sécurité ainsi que les partenaires extérieurs. C'est ce rapport qui, après l'élection d'Alpha Condé, servira de document de référence pour la préparation du processus de RSS dans le pays.

Les premières mesures de restructuration des FDS et la différence entre restructuration et réforme. Dès janvier 2010, après la signature de l'Accord de Ouagadougou, le général Sékouba Konaté commence un processus de vaste restructuration: imposition de la discipline et du respect de la hiérarchie militaire; lancement de grands chantiers de construction de casernes; fermeture du camp d'instruction de Kaléah (Forécariah) où se trouvent des miliciens; nouvelles nominations par décrets à la tête du haut commandement militaire; recrutement de 8000 élèves-gendarmes afin de sécuriser les frontières et le processus électoral.

Cette restructuration est nécessaire et elle est perçue positivement dans le pays mais elle ne représente qu'un premier pas en avant. Ces mesures dordre technique et professionnel visent à améliorer le fonctionnement, mais elles ne touchent pas encore aux causes des dysfonctionnements. Elles ont pour effet de montrer que la volonté politique est effective et que celle-ci va dans le sens 
de l'amélioration des conditions de vie et de travail des forces de défense et de sécurité, mais elles ne remettent pas en question le système. Par ailleurs, elles sont prises isolément au sein de la haute hiérarchie, sans tenir compte de léquilibre global des effectifs et sans processus participatif de la société civile.

Or, la réforme du secteur de la sécurité se définit comme une démarche à la fois politique et holistique. Elle a pour objectif l'instauration d'institutions de défense et de sécurité performantes, placées sous le contrôle d’organes légalement prévus par la constitution. Elle vise également létablissement d'une justice indépendante et forte. En principe, elle doit évoluer dans le cadre d'une concertation nationale. C’est ce type de réforme que le président Alpha Condé va tenter d'initier à son arrivée au pouvoir.

La mise en place et le rôle du CNT. Lordonnance portant création du Conseil national de transition (CNT) est signée par le général Sékouba Konaté le 9 février 2010. Le président de la transition nomme Hadja Rabiatou Serah Diallo, une responsable syndicale, à la tête de cette institution, sur proposition des Forces vives. Le 17 février, il lui adresse une lettre de mission dans laquelle il rappelle le délai de six mois à respecter pour l'organisation de lélection présidentielle. A cet effet, la présidente du CNT est invitée à procéder sans tarder à la composition de l'organe législatif de la transition (101 membres), dont le général indique les quotas par catégories. ${ }^{18}$ La liste des membres du CNT, nommés par décret présidentiel, est publiée le 7 mars. ${ }^{19}$

Finalement, la mise en place du CNT a lieu lors d'une cérémonie officielle d'installation, ponctuée du discours solennel du président de la transition (L'Observateur 2010: 2). Le CNT ne tarde pas à se mettre au travail. Une semaine après sa nomination, cet organe décide de créer neuf commissions réparties par domaines: révision constitutionnelle, relecture des textes électoraux, finances, traités et lois, évaluation de l'action gouvernementale, évaluation et suivi de la CENI, réconciliation nationale, défense et sécurité, communication. L'une des premières tâches du CNT est ainsi de procéder à la révision de la loi fondamentale et du code électoral pour permettre au pays d'aller aux élections avec une constitution et des textes adéquats.

La sécurisation $d u$ processus électoral. Contrairement à son prédécesseur, le général Sékouba Konaté a réussi, en tant que président de la transition, à mettre rapidement en place toutes les institutions nécessaires: le premier ministre de transition (Jean-Marie Doré, un leader de l'opposition), le gouvernement d'union nationale de transition, le Conseil national de transition et la CENI (présidée par Ben Sékou Sylla, l’ancien président du CNOSCG) ${ }^{20}$ Le général Sékouba Konaté a pris officiellement ses fonctions à la mi-janvier 2010 et le premier tour de lélection présidentielle a eu lieu six mois plus tard, le 27 juin. De plus, le président de la transition a assuré la sécurisation du processus électoral, avec la création par décret du 18 mai 2010 d'une force mixte, la Force spéciale de sécurisation du processus électoral (FOSSEPEL), commandée par létat-major mais placée sous l'autorité de la CENI. 
Létablissement du CNT et la sécurisation de lélection présidentielle ont abouti à un succès majeur et à une grande première en Guinée depuis l'indépendance: le premier tour d'une élection présidentielle démocratique, ouverte et transparente à laquelle aucun membre des institutions de la transition ne sétait porté candidat.

Malheureusement, le second tour du scrutin ne s'est tenu que le 7 novembre 2010. Il a été retardé par la composition d'alliances politiques autour des deux candidats restés en lice et par des calculs politiciens. L’entre-deux-tours a fait resurgir les "vieux démons» de l'exclusion et de la haine basés sur les appartenances ethniques et a donné lieu à des dérapages, de vives tensions et des violences. Le président de la transition, le général Sékouba Konaté, a même menacé de démissionner le 6 juillet 2010 face à des accusations de fraudes venant de l'Union des forces républicaines. L'incident a pu être réglé à temps. A l'issue de l'élection, c’est Alpha Condé qui a remporté le scrutin.

Lobjectif principal de la transition, qui consistait à mettre en place une gouvernance politique capable d'assurer le retour à l'ordre constitutionnel a été atteint. Pour y parvenir, le général Sékouba Konaté a dû faire preuve d'une volonté politique ferme et il a su tenir le cap. Cependant, le climat politique conflictuel qui a régné entre les deux tours de lélection présidentielle a laissé des traces profondes, qui vont avoir une incidence sur la gouvernance du nouveau chef de l'Etat.

\section{Le processus de changement depuis lélection d'Alpha Condé}

\section{Existence d'une volonté politique forte et d'une large consultation}

Dans son discours d'investiture, le 21 décembre 2010, le président Alpha Condé a fait de la réforme du secteur de la sécurité une des priorités de son programme de gouvernement et il a demandé l'appui des Nations Unies. A cet effet, dès janvier 2011, une mission de l'ONU dirigée par le général Lamine Cissé a élaboré les premiers documents techniques dans le prolongement des recommandations de l'évaluation réalisée en 2010; d'autres experts du Bureau de prévention des crises et du relèvement du Programme des Nations Unies pour le développement (PNUD), du Centre pour le contrôle démocratique des forces armées - Genève, du Bureau des Nations Unies pour l'Afrique de l'Ouest et du Département des operations de maintien de la paix des Nations Unies ont été déployés pour fournir une assistance technique au comité chargé d’organiser le future séminaire national sur la RSS. ${ }^{21}$

Le nouveau chef de l'Etat, qui est également chef des armées et ministre de la Défense, a confirmé sa volonté politique en inaugurant (et en clôturant), du 28 au 31 mars 2011, le Séminaire national sur la réforme du secteur de la sécurité. Un autre facteur important de réussite de ce séminaire a été la composition et 
le nombre élevé de participants qui ont réuni: le président de la République, le gouvernement, des institutions de l'Etat, des diplomates, des organisations internationales (en particulier l'ONU, l'UA, la CEDEAO), des organisations de la société civile, des pays invités, des commandements des régions militaires et des grandes unités de Conakry. Durant cette rencontre, les participants ont identifié les actions prioritaires et les séquences de mise en œuvre de la RSS à court (2011), moyen (2012) et long terme (2013-2015), secteur par secteur. Ces travaux ont fait lobjet d'un rapport qui a été publié.

Parallèlement, des états généraux de la justice, placés sous le haut patronage du président de la République, se sont tenus à Conakry du 28 au 30 mars 2011. Le rapport de synthèse a établi le diagnostic de la justice et a fixé trois objectifs: tout d’abord, l'indépendance de la justice par le biais de la réforme du Conseil supérieur de la magistrature (création d'organes disciplinaires, gestion du plan de carrière des magistrats, revalorisation salariale, statuts des greffes); ensuite, la construction et l'équipement de cours et tribunaux; enfin, la révision de la carte judiciaire (pour un meilleur accès à la justice) et de la carte pénitentiaire.

Le 14 avril 2011, le président de la République a signé le décret portant création du Comité national de pilotage de la RSS. Placé sous son autorité, il est chargé de la supervision, de lorientation politico-stratégique et du suivi de la mise en œuvre de la réforme. Il comprend : une Unité de gestion du programme, une Commission dorientation stratégique, une Commission technique de suivi et des Comités techniques sectoriels. Il s'agit là du premier pas franchi en vue de la mise en application de la réforme. Ce comité bénéficie du soutien de l'Etat guinéen et de l'appui du système des Nations Unies dans ce pays.

Objectifs partagés de la RSS. La réforme met l'accent sur la nouvelle conception de la défense et de la sécurité dans une approche globale, inclusive, consensuelle et participative qui repose sur l'implication de l'ensemble des acteurs et sur une appropriation nationale à tous les niveaux. Cette réforme s'inscrit au départ dans un élan de renouveau démocratique, d'instauration d'un véritable Etat de droit et vise à mettre en place un système judiciaire indépendant, impartial, compétent et accessible.

Lobjectif principal de la RSS est détablir un cadre institutionnel approprié, de doter l'appareil judiciaire et les forces de défense et de sécurité (armée, gendarmerie, police-renseignement, protection civile, douane, eaux et forêts, environnement) de capacités professionnelles et opérationnelles, de rapprocher ces services de la population, de permettre un contrôle civil et de garantir le respect des droits de l'homme. La réforme a également pour objectif de contribuer de manière transversale à la lutte contre la criminalité transnationale et contre la circulation des armes légères dans la région. Ces objectifs sont largement partagés par la population guinéenne.

Mise en ouvre de la RSS et rapprochement civilo-militaire. Les premiers pas de la réforme ont d'ailleurs été salués par les citoyens. Il s’est agi d’abord de sécuriser les populations civiles par le renforcement de la discipline au sein de l'armée, 
l'amélioration du comportement des militaires, le démantèlement des barrages et la démilitarisation de la capitale. Puis, des mesures de restructuration des forces en fonction d'impératifs de défense ont suivi: création de nouvelles unités, redéploiement et dispersion des militaires de l’ancienne garde présidentielle et du bataillon des troupes aéroportées (BATA) ou «bérets rouges». Enfin, des mesures de rapprochement entre FDS et citoyens ont été prises: redynamisation du Comité civilo-militaire et création du Service civique d'action pour le développement. En outre, les documents de base de la réforme, notamment les textes faisant partie du cadre juridique (textes législatifs tels que le code de justice militaire, le statut général et les statuts particuliers des militaires) et du cadre organisationnel des forces (attributions, emploi) ont été élaborés ou révisés par les services concernés.

La seconde phase de la réforme a consisté à définir un Plan d’action national pour la RSS. La méthode retenue a veillé à faire travailler chaque ministère, secteur par secteur, au niveau des cinq comités techniques sectoriels (défense, sécurité, justice, douane, environnement). A la mi-2012, ce travail a pu être réalisé: chaque secteur disposait d'un plan d'action sectoriel; depuis, les politiques sectorielles ont été validées par les différents ministres. Il reste à harmoniser et à consolider ce plan dans son ensemble, mais cette étape n’a pas encore eu lieu.

La RSS s'est développée en 2012 par la mise en œuvre de trois projets: recensement biométrique des militaires; mise à la retraite de 4000 militaires; et renforcement du contrôle démocratique civil des forces de défense et de sécurité. A cet effet, le PNUD a recruté trois experts chargés d'apporter des appuis techniques ponctuels. Ces projets ont été financés par le Fonds de la Commission de consolidation de la paix des Nations Unies (PBC 2012).

\section{Vers une Politique nationale de défense et de sécurité?}

Le 23 août 2013, c'est par la voix du ministre délégué à la Défense, maître Kabélé, que la Guinée a amorcé une nouvelle étape dans la RSS à travers le lancement d'une consultation nationale dans les régions, préfectures et communes du pays, en vue d'associer toutes les franges de la population à lélaboration de la future Politique nationale de défense et de sécurité. Ce processus est initié du bas vers le haut.

Entre-temps, le système des Nations Unies et les partenaires techniques et financiers continuent d'appuyer le processus de la RSS. L'Union européenne (UE) soutient, par le biais du 10e Fonds européen de développement, trois projets pilotes en faveur de la police de proximité, lobjectif étant de rapprocher les policiers et la population, de garantir un accès plus rapide des personnes à la sécurité, et de sensibiliser, sur le plan institutionnel, au contrôle et à la redevabilité de la police. De plus, l'UE appuie la protection civile (à travers la brigade des sapeurs-pompiers à Conakry) et le corps des gardes-forestiers dans les zones à 
risques (parc de Haute-Guinée, forêt classée de Ziama et aire protégée du Mont Nimba). ${ }^{22}$

Ces différents éléments montrent que, dans un premier temps, en 2011-2012, la RSS a pris un vrai départ et s'est développée avec succès du fait d'un consensus national sur ces questions. Mais cet élan va-t-il se maintenir?

\section{Le rôle des différents acteurs}

Différents types d'acteurs, internes et externes, institutionnels et plus rarement à titre individuel, jouent un rôle moteur en matière de RSS. Néanmoins, dans certains cas, la vigilance est de mise, car de grands défis restent à relever.

\section{Le gouvernement face au défi du dialogue politique et du respect des droits de l'homme}

Le pouvoir exécutif issu de l'élection présidentielle de novembre 2010 a fait preuve de volonté et d'ouverture face à la nécessaire réforme du secteur de la sécurité. Les jalons politiques pour une bonne gouvernance du secteur de la sécurité ont été posés.

Cependant, dans le contexte de la Guinée, une véritable RSS ne peut réussir que dans le cadre d'un Etat de droit démocratique qui n'est pas encore restauré dans ce pays. L'Etat de droit a été malmené par plus d'un demi-siècle de mauvaises pratiques. Bien que les institutions soient en place, leur fonctionnement laisse encore à désirer et le climat politique demeure tendu du fait de la manipulation ethnique et de la contestation des résultats de lélection présidentielle de 2010.

L'un des plus grands défis à relever pour ce gouvernement est le dialogue politique avec l'opposition. La méfiance reste forte entre les deux camps en raison des élections présidentielle et législatives passées. ${ }^{23}$ Ce n'est que le 28 septembre 2013, soit avec beaucoup de retard, que ce deuxième scrutin a pu avoir lieu. Aucune confiance ne règne entre les dirigeants au pouvoir et les leaders de l'opposition. Dès lors, le gouvernement actuel ne parvient pas à empêcher l'usage de la force en cas de manifestation, comme en témoigne, la mort d'un jeune élève survenue le 25 novembre 2013 au cours d'une marche de l'opposition qui contestait les résultats des législatives proclamés par la Cour suprême.

\section{$D u$ CNT à l'Assemblée nationale: finalisation de la transition et défis démocratiques}

Lélection des députés de l'Assemblée nationale est un grand pas en avant. Cette nouvelle assemblée élue démocratiquement va remplacer le CNT. Les résultats 
définitifs ont été proclamés par la Cour suprême le 25 novembre 2013: ils confirment les résultats provisoires qui avaient été publiés par la CENI le 18 octobre: sur les 114 sièges de l'Assemblée nationale, le parti d'Alpha Condé en remporte 53 et ses alliés 5 contre 56 pour l'opposition. La majorité au pouvoir l'emporte, néanmoins l’opposition est bien représentée.

Avec cette nouvelle assemblée, l'enjeu politique est de tourner la page de la période douloureuse de la transition, puisque le CNT était la dernière institution de la transition encore en vigueur après l'élection d'Alpha Condé. Il s'agit également pour la Guinée de se doter d'un véritable organe législatif ayant compétence pour contrôler l'action gouvernementale. En ce qui concerne la RSS, les nouveaux élus devront être sensibilisés ou formés au contrôle démocratique civil de l'action du gouvernement.

\section{Une Commission Réconciliation sans Vérité ni Justice}

Une Commission provisoire chargée de la réconciliation nationale a été créée par décret présidentiel le 15 août 2011 et deux hautes personnalités religieuses ont été nommées pour la coprésider. ${ }^{24}$ Néanmoins, la société civile ne semble pas avoir été suffisamment consultée au sujet de son mandat, de ses pouvoirs ni de sa composition. Alors que son rôle semble axé sur la réconciliation, qu'en est-il du volet «vérité » et du volet «justice», qui sont présents dans bon nombre de commissions vérité et réconciliation en Afrique et ailleurs dans le monde? La société civile souhaiterait que cette commission s'attaque à l'impunité et formule des recommandations en termes de poursuites judiciaires contre les auteurs présumés d’exactions.

Le programme d'action de cette commission provisoire comporte cinq étapes: la prière; lécoute; la réflexion basée sur les résultats du travail au niveau local, communal, préfectoral, national; la définition des orientations; et enfin la création d'un organe ou d'une institution chargé(e) de la réconciliation nationale.

En principe, après des consultations nationales et internationales, les coprésidents de la Commission provisoire chargée de la réconciliation nationale devraient faire des propositions en vue de la création d'une véritable commission vérité et réconciliation en Guinée. C’est ce que la société civile et certains leaders de l'opposition appellent de leurs vœux.

\section{Le médiateur de la République: un organe essentiel mais une personnalité controversée}

La Constitution de 2010 élaborée et adoptée par le CNT prévoit, en son titre $\mathrm{XI}$, la création de la fonction de médiateur de la République. Aux termes de l'article 127, le médiateur est « un organe intercesseur, gracieux et indépendant, entre l'administration et les administrés. Il reçoit dans les conditions fixées par 
la loi, les réclamations des administrés dans leurs relations avec les administrations de l'Etat, les circonscriptions territoriales, les collectivités locales, les établissements publics ainsi que tout organisme investi d'une mission de service public». Les modalités de saisine, d'intervention et de fonctionnement du médiateur de la République sont fixées par la loi.

Si la création de cette institution est à saluer, en revanche, il convient de s'interroger sur la personne choisie pour l'incarner. En effet, en janvier 2011, le président Alpha Condé a nommé comme médiateur de la République le général Faciné Touré. Ce dernier n’a pu prendre ses fonctions qu’en juillet, en raison d'une polémique soulevée par des propos qu'il a tenus et qui sont jugés inopportuns car porteurs de divisions sur des bases ethniques. Il importe de noter, de plus, que ce général est un ancien membre du Comité militaire de redressement national, le comité militaire au pouvoir au lendemain du coup d'Etat de Lansana Conté.

Il apparaît qu'une personnalité consensuelle, faisant preuve de neutralité et d'objectivité, n’ayant pas eu à exercer de fonction politique au cours des précédents régimes autoritaires et choisie pour ses compétences en matière de défense des droits de l'homme serait plus indiquée pour exercer une telle fonction. De tels profils existent dans la société civile.

\section{Le rôle des acteurs extérieurs}

Des acteurs extérieurs tels que le Groupe international de contact pour la Guinée (GIC-G) ont joué un rôle politique et diplomatique très important d'accompagnement de la transition en Guinée, tandis que d'autres, à titre individuel, comme le général Lamine Cissé ou encore les différents partenaires techniques et financiers, ont joué un rôle déterminant de conseil et d'assistance à la RSS.

Le GIC-G sest illustré par sa capacité à soutenir les acteurs de la transition, mais aussi à recadrer la feuille de route de la transition et à prendre des sanctions lorsqu'il constatait des dangers pour la paix et la sécurité du pays et des risques de violations des droits de l'homme. Ce groupe a été créé par la communauté internationale en janvier 2009 à Addis Abeba, en marge d'une réunion del'Union africaine. ${ }^{25} \mathrm{Il}$ sest réuni régulièrement, à 17 reprises, durant deux ans entre février 2009 et février 2011. La dernière réunion, qui a mis fin à son mandat et ouvert la possibilité de la création d'un groupe des amis de la Guinée, a eu lieu à Conakry le 10 février 2011, quelques semaines après la prise de fonctions du nouveau président élu.

Le général Lamine Cissé est un officier sénégalais à la retraite, qui est intervenu avec succès en Guinée plusieurs fois à partir de 2010 pour conseiller, initier, coordonner et faire le suivi de la RSS. Il jouit d'une grande notoriété, en tant notamment qu'ancien ministre de l'Intérieur dans son pays mais aussi en tant qu’ancien représentant du Secrétaire général des Nations Unies et chef du Bureau de l'Organisation des Nations Unies en République centrafricaine. 
Sa longue expérience, son sens du contact et son excellente connaissance du milieu ouest-africain ont été des atouts pour sa mission.

Les partenaires techniques et financiers sont les principaux partenaires bilatéraux et multilatéraux au développement de la Guinée qui appuient la RSS. Il s'agit notamment de la France, des Etats-Unis, de l'Union européenne et de l'Organisation internationale de la Francophonie. Ces acteurs extérieurs envoient sur place des experts civils et/ou militaires afin de soutenir, respectivement: le Service civique d'action de développement, le renforcement des capacités institutionnelles en matière de sécurité et de RSS, la réforme de la justice, la formation de cadres militaires en matière de respect des droits de l'homme.

\section{Conclusion}

La question qui se pose à présent est de savoir si le changement va se poursuivre. On se souvient que la RSS était devenue une impérieuse nécessité en Guinée en raison d'une gouvernance conflictuelle et violente depuis l'indépendance. Cependant, la RSS n'avait pas été possible sous Lansana Conté car ce dernier empêchait toute velléité de réforme. Il en a été de même sous Moussa Dadis Camara. Les premiers changements annonciateurs d'une transformation de la gouvernance du secteur de la sécurité et d'une RSS ont été introduits par le général Sékouba Konaté. Dans un contexte politique favorable, celui-ci fait preuve d'une volonté politique sans faille pour initier la restructuration de l'armée, donner son accord à lévaluation du secteur de la sécurité et mener à terme le processus électoral permettant un retour à l'ordre constitutionnel. Ces conditions, indispensables pour lancer un véritable processus de RSS, ont été réunies à ce moment-là.

Les deux premières années de la gouvernance d'Alpha Condé ont été très prometteuses en matière de gouvernance du secteur de la sécurité et de reforme. La volonté politique forte, la recherche douverture et de consensus national, l'appropriation et la mise en œuvre qui ont suivi, du début de l'année 2011 à fin 2012, ont été les premiers pas d'une véritable transformation.

Cependant, tout au long de l'année 2013, ce processus n’a pas avancé. La principale raison de ce blocage est le climat politique conflictuel entre la mouvance présidentielle et lopposition et les violences qui ont émaillé la préparation, la tenue et la proclamation des résultats des élections législatives. En outre, le fait de commencer la réflexion sur la Politique nationale de défense et de sécurité par une consultation au niveau local et préfectoral peut aussi être interprété comme une manière de vouloir temporiser et gagner du temps. L'immobilisme est peut-être une option gouvernementale, en attendant de reprendre la réforme là où elle s'est, semble-t-il, arrêtée.

L’engouement du départ est donc retombé. Le risque serait de ne plus accorder par la suite d'attention suffisante à la réforme du secteur de la sécurité. Or, 
la réforme, et en l'occurrence le Plan d'action national pour la RSS, est encore à adopter, à programmer, à chiffrer et à mettre en œuvre. Tout un programme ...

\section{Notes}

${ }^{1}$ Après la rupture avec la France, le président Sékou Touré se tourne vers le bloc soviétique et entame une coopération civile et militaire avec Moscou et d'autres alliés des pays de l'Est.

2 «Sékou Touré voulait une armée docile, au service de son pouvoir, prête à exécuter ses ordres sans état d'âme. Pour lui, l'armée, le parti, tout ce qui existe en Guinée devait contribuer à son rayonnement personnel» (Bah 2009: 421).

${ }^{3}$ Sékou Touré retire à l’armée le Deuxième Bureau (renseignements), préférant confier les Services spéciaux à son frère Siaka Touré.

${ }^{4}$ Selon CAMARA Kaba 41 (1998: 64), la prison appelée «camp Boiro» a été construite en 1961- 62 par les Tchèques sous l'égide de Keita Fodéba, ministre de l'Intérieur et de la Sécurité et en même temps ministre de l'Armée populaire guinéenne.

${ }^{5}$ Malgré sa soumission au pouvoir, l'armée néchappera pas à la tyrannie: «contrainte d’obéir aveuglément aux ordres d’un seul homme, larmée a servi la volonté de puissance ... du leader du PDG tout au long des vingt-six ans de la révolution.» Toutefois, «les chefs militaires subiront le même sort que les victimes qu'ils avaient torturées et tuées avant leur disgrâce» (Bah 2009: 153).

${ }^{6}$ Larticle 34 de la Constitution stipule que: «En cas de vacance de la fonction de président de la République consécutive au décès ou à la démission du président de la République ..., la suppléance est assurée par le président de l'Assemblée nationale ou, en cas d'empêchement de celui-ci, par l'un des vice-présidents. La vacance est constatée par la Cour suprême, saisie par le président de l'Assemblée nationale ...».

7 Adopté le 14 janvier 2009, ce décret nomme les membres du gouvernement (Guinée 2009).

${ }^{8}$ Cette loi ne faisait pas l'unanimité car les défenseurs des droits de l'homme et les représentants des victimes réclamaient une Commission d'enquête indépendante, si possible internationale.

${ }^{9}$ Accord signé le 27 janvier 2007 par les syndicats, le patronat et le gouvernement intitulé: Procès-verbal de négociations suite à la grève générale de l'Inter centrale syndicale CNTG-USTG élargie à l'ONSLG et à l'UDTG déclenchée le 10 janvier 2007.

${ }^{10}$ Après le premier tour de lélection présidentielle de juin 2010.

${ }^{11}$ Selon l'article 146: «L'Institution nationale indépendante des droits humains est chargée de la promotion et de la protection des droits humains». Selon 
l'article 148: «Une loi organique fixe la composition, l'organisation et le fonctionnement de l'institution".

${ }^{12}$ Ces appellations rappellent les institutions françaises: en France, «la Commission nationale consultative des droits de l'homme est l'Institution nationale des droits de l'homme créée en 1947 ». En Afrique, les institutions nationales indépendantes des droits humains sont des organes statutaires établis par les gouvernements; ils ont la responsabilité de promouvoir et protéger les droits de l'homme dans leurs pays respectifs. La création et le fonctionnement de ces institutions doivent être conformes aux Principes des Nations Unies pour la protection et la promotion des droits de l'homme (Principes de Paris). Les institutions nationales indépendantes des droits humains sont également tenues d'assister la Commission africaine des droits de l'homme et des peuples. En Afrique, plusieurs Etats (Afrique du Sud, Cameroun, Mali, Maurice, Mauritanie, Niger, Rwanda, Sierra Leone, Togo ...) disposent d'une CNDH (CADHP 2015).

${ }^{13}$ Le Peace Building Fund est un fonds de consolidation de la paix qui finance les projets de la Commission de consolidation de la paix des Nations Unies (New York).

${ }^{14}$ Le coup d'Etat de décembre 2008 a été perpétré par le capitaine Moussa Dadis Camara, alors en poste au Service des carburants de l'armée, et le colonel Sékouba Konaté, commandant du BATA, une unité d'élite. M. D. Camara a également servi dans le BATA auparavant. Les deux hommes se connaissent bien et s’apprécient. M.D. Camara nomme Sékouba Konaté au grade de général en janvier 2009.

15 Moussa Dadis Camara a d'abord été évacué et soigné au Maroc. Puis, dans la soirée du 12 janvier 2010, il est arrivé au Burkina Faso, où se trouve le médiateur de la crise guinéenne, pour poursuivre son rétablissement.

${ }^{16}$ Le général Sékouba Konaté peut se permettre cette condamnation car, au moment des faits, le 28 septembre 2009, il était en mission à l'étranger. Il n’a donc pas pris part aux massacres.

17 Ont également pris part à cette mission conjointe: les partenaires bilatéraux et internationaux tels que la France et les Etats-Unis, l'Union européenne et l'Organisation internationale de la Francophonie.

${ }^{18}$ Le nombre de 101 membres est prévu par la Déclaration conjointe de Ouagadougou. Le détail, fixé dans la Lettre de mission, est le suivant: 30 (partis), 15 (syndicats), 9 (société civile), 8 (extérieur), 3 (ordres professionnels), 4 (coordinations régionales), 10 (forces de défense et de sécurité), 2 (droits de l'homme), 4 (jeunes), 3 (patronat), 3 (médias privés), 4 (femmes), 2 (org. paysanes), 2 (org. artisanales), 2 (congrégations religieuses).

19 C'est avec surprise que les citoyens guinéens constatent un dépassement du nombre des membres, qui est passé de 101 à 155 (Guinée 2010b).

${ }^{20}$ Jean-Marie Doré a été nommé premier ministre par décret du général Sékouba Konaté le 19 janvier 2010 et il a pris ses fonctions le 26 janvier à 
l'issue d'une cérémonie de passation de pouvoir avec Kabiné Komara. Dans un bref discours, le nouveau premier ministre a indiqué que ses deux missions fondamentales concerneront les élections et la refonte de l'armée, une façon de montrer qu'il a bien compris le sens de son devoir à la tête du nouveau gouvernement de transition.

${ }^{21}$ Le séminaire national sur la RSS est organisé sur financement du PNUD avec l'appui technique et logistique du système des Nations Unies en Guinée.

${ }^{22}$ Voir, par exemple, le communiqué de presse de la Délégation de l'Union européenne en Guinée (2013).

${ }^{23}$ Plusieurs forums de dialogue politique ont été organisés avec le soutien de la communauté internationale (ONU-CEDEAO-l'Organisation internationale de la Francophonie-UE-France-Etats-Unis): le Dialogue politique inclusif qui sest tenu à Conakry du 27 décembre 2011 au 22 février 2012; puis le Dialogue inter-guinéen qui sest tenu à Conakry du 5 au 9 juin 2013 puis les 2 et 3 juillet 2013 en vue de trouver un accord pour la préparation et la tenue des élections législatives.

${ }^{24}$ Il s'agit de Mgr Vincent Koulibaly, archevêque de Conakry et d'El Hadj Mamadou Saliou Camara, Imam de la mosquée Fayçal à Conakry.

${ }^{25}$ Le GIC-G comprenait: les Nations Unies, l'Union africaine, l'Union européenne, l'Organisation internationale de la Francophonie, la Communauté économique des Etats de l'Afrique de l'Ouest (CEDEAO), l'Union des Etats du Fleuve Mano, l'Organisation de la Conférence islamique, la Communauté des Etats sahélo-sahéliens, la Banque mondiale, l’Angola (pour la présidence du Conseil de paix et de sécurité de l'Union africaine), le Nigéria (en qualité de président de la CEDEAO), ainsi que des Etats africains (le Burkina Faso chargé de la médiation) et des membres permanents du Conseil de sécurité des Nations Unies (le Royaume-Uni, la France, la Russie et les Etats-Unis d'Amérique), l'Espagne, puis le Maroc (janvier 2010). La Côte d'Ivoire, le Ghana, la Guinée-Bissau, le Mali et le Sénégal ont également pris part à ce groupe. 